\title{
The Design of Automatic Temperature Control System for the Dyeing Machine
}

\author{
Jing Dai \\ Institute of Computer Technology \& Automatization \\ Tianjin Polytechnic University \\ Tianjin 300160, China \\ E-mail: mandy_jing@163.com
}

\begin{abstract}
This article analyzed the temperature control system of the dyeing machine in the textile industry, adopted the technology of PLC to automatically control the temperature and realized the automatic temperature adjustment in the work process of the dyeing machine. The spot adjustment proved that this method could fulfill the basic requirement of the dyeing machine to the temperature.
\end{abstract}

Keywords: PLC, Dyeing machine, Temperature

\section{The temperature control principle of the dyeing machine}

\subsection{The application of automatization technology in the textile industry}

The textile machinery manufacturing of China has been quickly developed for almost twenty years and has acquired great achievements, and the technical level and product stability of textile machinery have been fully enhanced. The automatic control of the textile machinery takes the sports control as its core, and is assisted by the textile technical parameters. In recent ten years, the electric power electronic technology, computer control technology and network communication technology have been applied and extended in the control and production management of the textile machinery quickly, which make the mechanical and electronic integration level of China fully enhanced.

\subsection{The intention of the control}

The parameters of the textile technology are numerous, which generally includes temperature, pressure, flux, liquid position, length, speed and displacement. The dyeing technology of the textile has strict requirement of the temperature, and the temperature ascending process, temperature keeping process, temperature descending process of the dyeing trough must accord with the technical requirements, and the manual control is very difficult. If the used machine is the small sample machine, it may bring trouble for the bulk samples. And the production equipments will make the color replay difficultly and induce serious kettle difference, and finally bring unnecessary losses for the production.

Therefore, the automatic temperature control of the dyeing machine is very important. The control system is required to freely set up the temperature ascending curve, temperature keeping curve, temperature descending curve, and the operation time, actual temperature and process temperature in the work process can be displayed to ensure the right implementation of the technology.

\subsection{The Structure of the temperature system}

The control objective is the dyeing sample machine, and the structure of its temperature system is seen in Figure 1. The temperature control range of the system is from 20 to 150 centigrade degree, and because the water can not achieve 150 centigrade degree, so the glycerin is adopted. The glycerin can achieve 150 centigrade degree under normal pressure.

When the dyeing machine is working, the temperature of the glycerin in the dyeing trough must be changed according to the technical requirements. If the temperature is lower than the set value, the temperature control system should close the heat switch 7, heat glycerin through the heating wire and stop heating until the temperature achieves the set value. If the temperature of the glycerin is higher than the set value, i.e. the excessive adjustment occurs, or the equipment is working in the quick descending stage of the temperature, the temperature control system will connect the cooling valve 8 to drop the temperature of the glycerin.

Common temperature controller can implement temperature keeping control, which is used in some prior dyeing 
machines, and if the automatic degree is further enhanced to make the equipment ascend, keep and descend the temperature according to needed speed until the whole technical process ends, the simple temperature control can not fulfill these requirements, and it is very necessary to design a sort of reliable, convenient temperature complete automatic control system with perfect functions.

\section{The design of the temperature control system}

\subsection{The total structure of the system}

In the industry, there are many methods to control the temperature, and here we select PLC to control the temperature. Figure 2 is the sketchy structure of the temperature control system.

The set parameters can be input into PLC through the human-computer interface, and PLC inputs the actual temperature into the $\mathrm{A} / \mathrm{D}$ convertor through the temperature sensor and a series of automatic computation, and the $\mathrm{A} / \mathrm{D}$ convertor transforms the temperature and inputs it into the PLC. Through analysis and computation, PLC can exactly decide open the heating switch or the electromagnetic valve.

\subsection{The PLC control method}

The typical temperature process curve of the dyeing machine is seen in Figure 3 which shows the temperature linearly changed with the subsection. Only the operator sets up the temperature and completed time required by the intersection points of various subsections such as $1,2,3 \ldots$. PLC will automatically compute the corresponding time and temperature value of all points on the curve. After the dyeing machine starts, PLC continually compares the actual temperature in the glycerin trough with the corresponding set temperature on the curve to decide whether the output state is heating, keeping or dropping temperature.

The ideal temperature change in the dyeing process is seen in Figure 4.

When PLC computes the temperature, it divides every temperature sect into many corresponding little interval according to the time, which is called as "step", and PLC computes the corresponding temperature of every step and take it as the set temperature value in this step, so the actual temperature curve provided by PLC is trapeziform, which is seen in Figure 5.

If only the time of every step is enough small, the difference between the computed temperature value and the ideal value in one step can be neglected, and because the heat capacity of the glycerin trough is large, so the system has certain heat inertia. So the temperature ascending curve in the trough will change according to Figure 6, not trapezifrom or approximate exponential curve, which more closes ideal temperature ascending curve with equal speed. The step length is smaller, the control precision is higher, but the performance of the power control circuit should be considered at the same time. The heating wire of the sample machine adopts the contactor to realize connection or breaking, so the frequency is not allowed to be higher, and the step length is confirmed as 6s. If the bidirectional crystal brake-tube or high power transistor is used, the step length can be largely reduced, and we only need considering the limitation of the shortest scanning cycle of PLC.

According to above control principle, we divide the whole work process into many sects, and every sect is divided into many steps, and PLC computes the output state of this step to automatically control the temperature.

\section{The selection of hardware}

\subsection{The selection of PLC}

The PLC of new generation was pushed out in the late of 1995 by Germany Siemens Company, which was not only improved in many aspects such as instruction system, operation speed and machine structure comparing with former S5 series, but largely reduced in the price and even achieved higher level than other brands with same functions, and its abundant CPU types and level classes make it possess strong adaptability when solving the problems of users' industrial automatization. Therefore, we select the S7-200 series PLC.

It is a sort of waste to blindly select the machine type with more point number. The input signals of the PLC control system in this project include start switch, pause switch and stop switch for the dyeing machine. The output of PLC mainly includes heating switch, cooling water valve switch, temperature ascending display lamp, temperature keeping display lamp and temperature dropping display lamp. So S7214 can fulfill these requirements. Figure 7 shows the automatic temperature control system of PLC.

\subsection{The selection of A/D transformation module}

We select EM325 as the A/D conversion module which simulation input/output is 4input/1output $\times 12$ bits.

\subsection{The selection of human-computer interface}

For the human-computer interface, we use TD200 which is professionally designed for S7PLC, and it has liquid crystal display screen which can display two rows or 80 characters or numbers, and TD200 has 9 input keys which can 
complete parameter display, data input and modification, the start and stop of data, and can be used as the machine operation panel.

The work program of TD200 is called as parameter module which is stored in the EEPROM of CPU of PLC. The contents that TD200 display are called as information. The programming of parameter module and information and the main program programming of PLC are implemented by the S7 programming software on the computer.

\section{The programming of PLC}

\subsection{The programming figure of $P L C$}

The whole work process confirmed in this article is divided into many sects, and every sect is divided into many steps according to the same time (6s), and PLC computes the output sate of this step to achieve the intention of automatic control. The main program figure of PLC control is seen in Figure 8.

From Figure 8, we can see that the program is mainly divided into two loops. The interior loop program includes the programs to compute the corresponding temperature on the typical temperature process curve of the dyeing machine, read A/D filtering wave, and judge ascending, keeping and dropping temperature. The exterior loop program mainly charges for reading the data of next sect when the present sect ends, and judging whether the total program operation is completed.

\subsection{The programming of PLC}

First, store the set data into the memorizer $\mathrm{V}$, store the original time 0 into VW0, and store the room temperature into VW2. Store the time from the origin to the point of 1 into VW4, and store the temperature that the point of 1 confirms in advance into VW6. Store the values of points of 2, 3, 4 and 5 into VW8-VW22 in turn. Store the set sect number into VW32. Establish two fingers, $\mathrm{AC} 1$ and $\mathrm{AC} 2$, and appoint their addresses to the start value and terminal value of every sect, and add 4 to them respectively after every sect completes operation, so they will be appointed to the address of the next sect and the intention of the cycle is achieved.

When every sect starts, the computation is implemented first. Take out the values of the storage units that AC1 and AC2 point and store them into the appointed units (VW24-VW30), and then compute how many steps that the sect can be divided into and how many centigrade degrees of the start and end points in the required time, and store them respectively into fixed storage units.

The number of the step and the temperature difference in this sect can help us to compute the concrete values that every step ascends or descends temperature. Use timers T101 and T102, and in every 6s, they time the time alternately and T102 produces a pulse.

From the first step, when T102 is connected, it will gradually add the changeable temperature value of every step on the start temperature, so the temperature value on the corresponding curve will be obtained, i.e. the required temperature value.

The timer T102 connects once and the corresponding curve value will change once and compare with the actual temperature value of the glycerin. If actual temperature is higher, the cooling water valve will be connected and the temperature dropping display lamp is lightened, and if the corresponding curve temperature is higher, the switch of the heating wire will be connected and the heating wire heats the glycerin and the temperature ascending display lamp is lightened, and if both is same, only the temperature keeping display lamp is lightened. So the control to the glycerin temperature is achieved.

After one sect operation is completed, the value of the finger is automatic increased and the sect number stored in VW32 reduces 1 . Then operate next sect, recompute the values, repeat the works of the previous sect, and implement from one sect to another in this way until the value of VW32 is 0 , so the control work is completed.

In order to reduce the times to start the cooling valve and drop the temperature only when the actual temperature exceeds the corresponding curve temperature, set up a temperature value, connect the cooling valve only when the actual temperature exceeds the corresponding curve temperature and this temperature value. In the program, subtract the value of the corresponding curve temperature from the actual temperature value, and store the computation result into VW62, and connect the cooling water valve to drop the temperature when VW62 exceeds the set value of the temperature.

To ensure the normal operation of PLC and protect PLC at the same time, compulsively transform to the mode of STOP when $\mathrm{I} / \mathrm{O}$ error happens.

\section{The simulation experiment}

\subsection{The running of the program}

First program by means of the design software of STEP 7-Micro/Win 32, and use LAD as the programming language. 
After program, load the program to the Siemens 214PLC under the mode of STOP, and transform it from the mode of STOP to the mode of RUN, and the program can be run. In the experiment, various parameters can be set in advance and input into PLC. According to appointed condition, PLC can automatically decide whether to ascend, keep or drop the temperature.

In order to set up the start temperature in 5 centigrade degree when the experiment runs in the first sect, the temperature of 1 point is appointed as 15 centigrade degree and the time is 1 minute, that is to say, the glycerin temperature in the first sect is required to ascend 10 centigrade degree. The time of the first sect is 60 seconds, and 1 step needs 6 seconds and is divided into 10 steps, so every step needs to ascend 1 centigrade degree. Then set up to drop the temperature when the actual temperature exceeds the curve temperature 1 centigrade degree. After these data are set up, and PLC can automatically and exactly compute various values.

First suppose that the actual temperature of the glycerin is changeless and its value is 8 centigrade degree. After the PLC switch is transformed to the mode of RUN, the parameters will be automatically input into PLC and stored. Then connect I0.0, set up M1.0, and the program formally begins to run. PLC implements one computation in every 6 seconds and judge the first 6 seconds and lightens Q0.3, and it exports nothing. After 6 seconds, if the actual temperature is higher, so lighten Q0.1 and Q0.4. The corresponding curve temperature continually ascends, and when its value achieves 7 centigrade degree, the actual temperature only exceeds 1 second, so only I0.3 is lightened, and in next 6 seconds, the actual temperature and the curve temperature are same and the output is changeless. After that, the curve temperature continually ascends, Q 0.0 and Q0.2 are lightened. The program will be run all along until it ends.

\subsection{The use of $A / D$ converter}

To change the actual temperature, we use EM235.

In actual production, we need to input the temperature of the glycerin into the $\mathrm{A} / \mathrm{D}$ convertor by the temperature sensor, and input the temperature into the PLC through the A/D convertor. For the temperature sensor, we can select PT100 which is platinum resistance temperature sensor, and it can measure the temperature between -60 centigrade degree to +400 centigrade degree.

In order to measure the temperature by EM235, we can connect PT100 temperature sensor on one input channel of the module. To transform the resistance that reflects the temperature change of PT100 to the voltage, the simulation output is used as the constant current source, i.e. export the constant current of $12.5 \mathrm{~mA}$ and supply it to the sensor of PT100. In this circuit, the linear input voltage of $5 \mathrm{mV} / \mathrm{C}$ is produced. EM235 transforms this voltage to the digital quantity, and then the program reads these values periodically and computes the temperature by these values. In the experiment, we can connect a potentiometer in the module EM235, so we can properly adjust the potentiometer to change the actual temperature which is input into PLC and make the experiment achieve the anticipated intention.

\subsection{The human-machine interface}

In the production, the value of the start point on every sect can be input through the human-computer interface of TD200 which implements programming by the software of STEP 7-Micor/Win and doesn't need other parameter evaluating software. One special area in the CPU of S7-200 series is reserved to change data with TD200. TD200 can interviews the necessary functions of CPU through the data area.

Connect TD200 with PLC, which can conveniently input and modify various parameters, and can also display the operation time and actual temperature in the production process to ensure normal operation of the technology.

\section{Conclusions}

Through operating the program on Siemens S7-200PLC, the actual temperature will occur in different time sects, so the outputs in PLC will be different, and the PLC can exactly judge whether to ascend the temperature, keep the temperature or drop the temperature and display which display lamp exactly according to the actual temperature and the computed corresponding curve temperature. After EM235 is extended, the adjustment of the value of the potentiometer can realize anticipated objective, which indicates that the PLC can basically realize the automatic control to the temperature system of the dyeing machine in actual production, and this method is feasible. And because Siemens S7 series is improved in many aspects such as instruction system, operation speed and machine structure than former products, and its price is low, so this method is also feasible for the economy.

\section{References}

Gao, Xiaogang. (2005). The Application and Development of Textile Machinery Automatization Technology. Textile Machinery. No.6. 8(4).

Song, Jianping. (2005). The Technical Innovation and Diversification Strategy of Textile Machinery Manufacturing. Textile Machinery. No.6. 2(1).

Yuebin. (2005). The Application of S7-200 to False-twist Texturing Machine. Textile Machinery. No.1. 




Figure 1. The Temperature System of the Dyeing Sample Machine

Note: 1- dyeing trough, 2- glycerin, 3- heating wire, 4- cooling water pipe, 5- mixer machine, 6- temperature sensor, 7heating switch, 8 - cooling water valve.

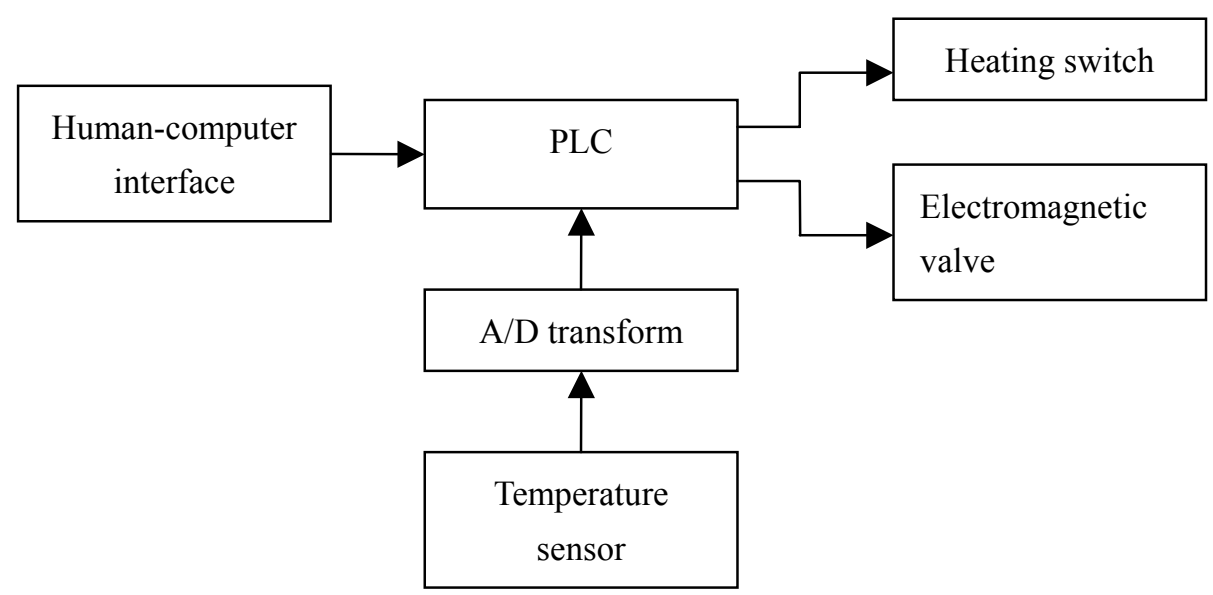

Figure 2. The Sketchy Structure of Control System

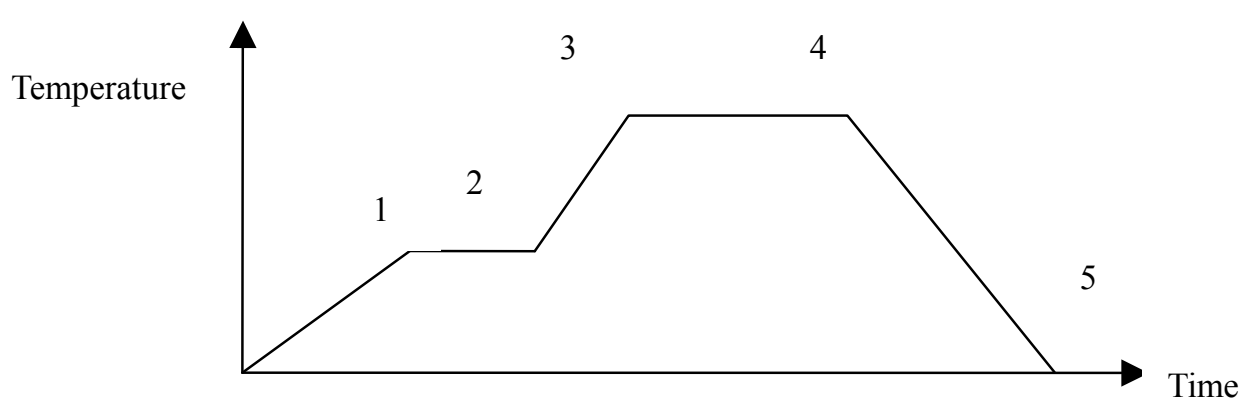

Figure 3. Typical Temperature Process Curve of the Dyeing Machine 


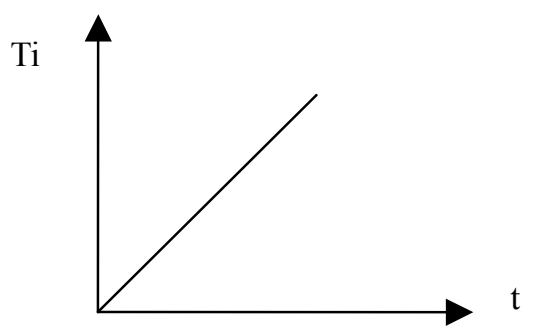

Figure 4. Ideal Temperature Process

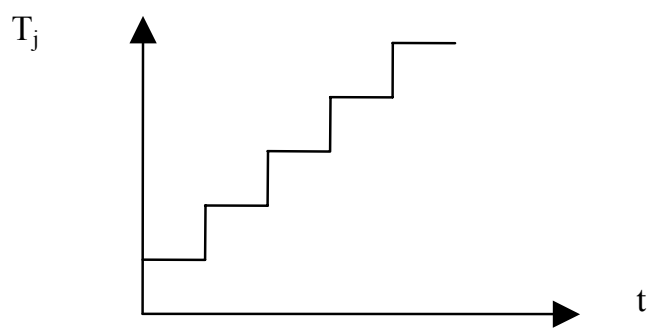

Figure 5. PLC Interpolation Temperature $\mathrm{Tj}$

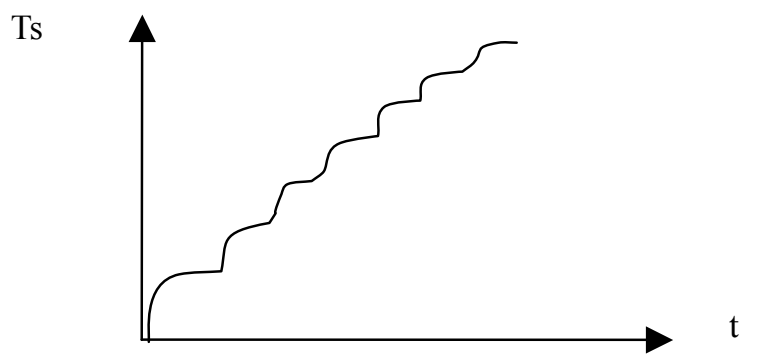

Figure 6. Actual Temperature of the Dyeing Trough Ts 


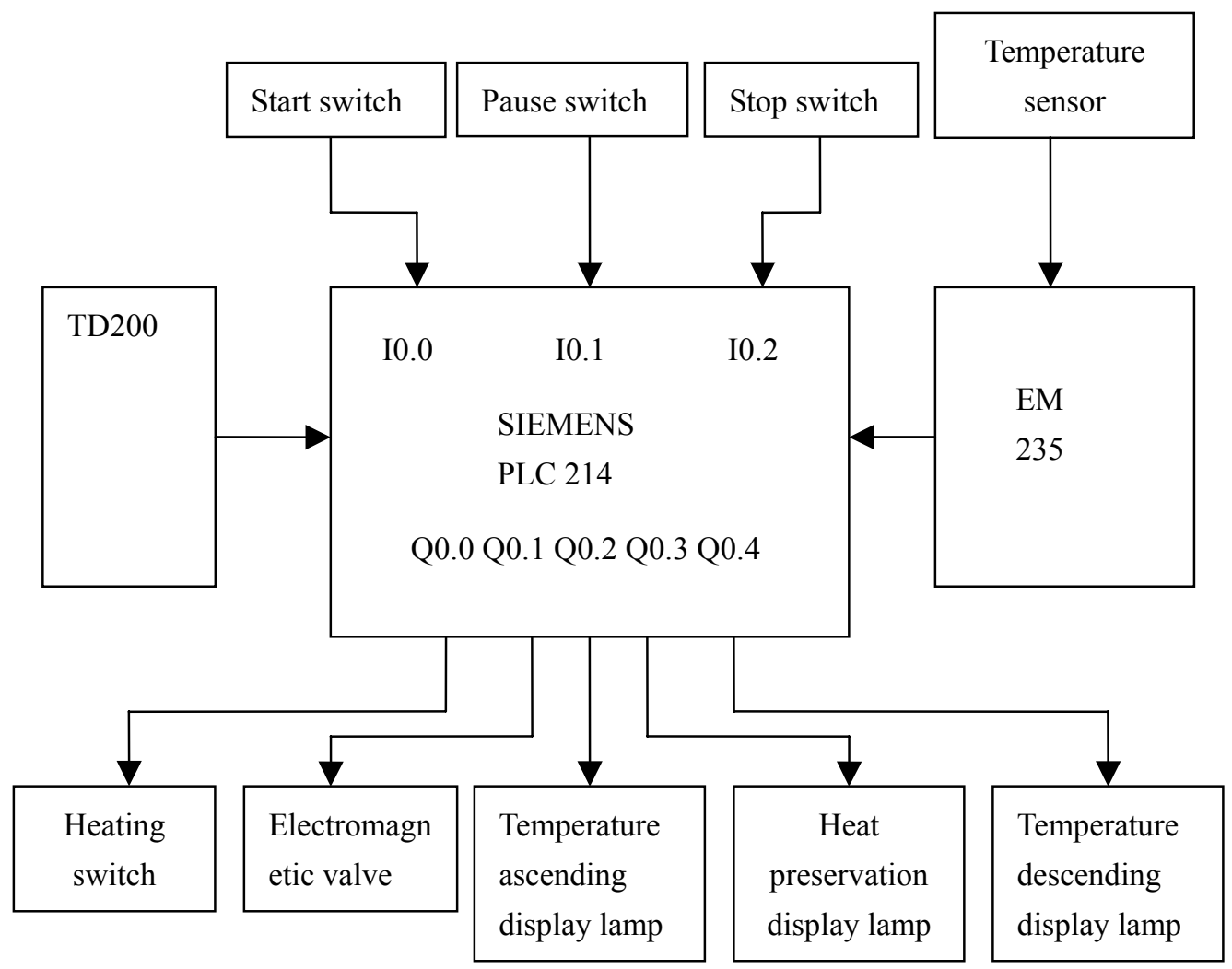

Figure 7. S7 PLC Automatic Temperature Control System 




Figure 8. Main Program Layout 